\title{
Catalyst Composition and Content Effects on the Synthesis of Single-Walled Carbon Nanotubes by Arc Discharge
}

\author{
Tingkai Zhao, ${ }^{1,2}$ Qinghu Tang, ${ }^{3}$ Yongning Liu, ${ }^{1}$ Changchun Zhu, ${ }^{2}$ and Xiang Zhao ${ }^{4}$ \\ ${ }^{1}$ State Key Laboratory for Mechanical Behavior of Materials, Xi'an Jiaotong University, Xi'an 710049, China \\ ${ }^{2}$ School of Electronics and Information Engineering, Xi'an Jiaotong University, Xi'an 710049, China \\ ${ }^{3}$ School of Energy and Power Engineering, Xi'an Jiaotong University, Xi'an 710049, China \\ ${ }^{4}$ School of Science, Xi'an Jiaotong University, Xi'an 710049, China
}

Received 15 January 2007; Accepted 16 March 2007

Recommended by Rakesh K. Joshi

\begin{abstract}
Single-walled carbon nanotubes (SWCNTs) were prepared by a modified arc discharging furnace using Fe-Ni-Mg powders as catalyst at $600^{\circ} \mathrm{C}$. The effects of catalyst composition and content on the production rate and purity of SWCNTs are investigated in this paper. When the Fe-Ni-Mg catalyst composition is $2: 1: 2 \mathrm{wt} \%$ and the catalyst content is $5 \mathrm{wt} \%$, the experimental results indicate that the production of SWCNTs is 12 grams per hour, and the purity and diameter of SWCNTs are $70 \%$ and 1.22 $\sim 1.38 \mathrm{~nm}$, respectively. The results indicate that the cooperative function of catalyst composition and content plays an important role in the production of SWCNTs. The aim of this work is to control the production process of SWCNTs efficiently.
\end{abstract}

Copyright (c) 2007 Tingkai Zhao et al. This is an open access article distributed under the Creative Commons Attribution License, which permits unrestricted use, distribution, and reproduction in any medium, provided the original work is properly cited.

\section{INTRODUCTION}

Since the discovery of single-walled carbon nanotubes (SWCNTs) by Iijima and Bethune in 1993 [1, 2], there has promised a new field of science and technology with their specially elongated fullerene structure. But the research and application of SWCNTs have been confined in a considerable degree because of low production rate. Therefore, how to improve the production rate of SWCNTs has become a difficult work. Many researchers have carried out large amounts of significant studies on this area [2-7]. There are mainly three of synthesis methods, such as arc discharge (AD) [8], laser ablation (LA) [9], and chemical vapor deposition (CVD) [10].

For all methods, catalyst plays a very important role on the production of SWCNTs. Generally, a metal or an alloy powder as catalyst is necessary for the growth of the SWCNTs according to the growth mechanism. A suitable catalyst and other conditions can improve the production of SWCNTs. Therefore, the investigation of catalyst including its type, composition, and content is very important to control the synthesis of SWCNTs on large scale. Generally, SWCNTs are prepared by using catalysts which include transition single metal or multimetals, such as $\mathrm{Fe}, \mathrm{Co}$, and $\mathrm{Ni}$ or $\mathrm{Co} / \mathrm{Ni}$ and $\mathrm{Ni} / \mathrm{Y}$ [11-14]. All the catalysts are effective to improve the production of SWCNTs under certain conditions. According to previous literatures $[6,7], \mathrm{Fe}-\mathrm{Ni}-\mathrm{Mg}$ powder as catalyst is one of the more efficient catalysts to prepare SWCNTs by arc discharging furnace at controlled temperature on large scale and high purity at present. The effect of temperature on the formation of SWCNTs has been studied in detail [6]. However, the effects of catalyst composition and content on the growth of SWCNTs are not well investigated in previous paper. Therefore, it is necessary to carry out this research continuously and deeply so that we can commendably control the formation of SWCNTs. The aim of this work is to develop a control method which can synthesize SWCNTs on large scale and high purity so that they meet the needs of researches and applications.

\section{EXPERIMENTAL}

In our experiment, SWCNTs were prepared by arc discharging furnace at controlled temperature using a mixture of Fe$\mathrm{Ni}-\mathrm{Mg}$ powders as catalyst at $600^{\circ} \mathrm{C}[15]$. The arcing current is $100 \mathrm{~A}$ and the static helium atmosphere pressure is 500 torr. Finally, we obtained a cloth-like soot formed on the entire inner wall of the chamber and, in general, an $80 \mathrm{~mm}$ anode rod is used up in 10 minutes and $2 \mathrm{~g}$ of soot can be collected. 
The catalyst powder was mixed using $\mathrm{Fe}, \mathrm{Ni}$, and $\mathrm{Mg}$ (all the chemical purity) elements. Then the powders were ballmilled by planetary ball miller about $30 \mathrm{~min}$.

The characterization of SWCNTs were studied by using SEM(JEOL JSM-6700F), TEM(JEOL JEM-200CX), HRTEM(JEOL JEM-2010), XRD(RIGAKU D/MAX-2400, with $\mathrm{CuK}_{\alpha}$ ), and Raman scattering spectroscopy(Raman 950, with wavelength $1064 \mathrm{~nm}$ ).

\section{RESULTS AND DISCUSSION}

Under the fixed conditions, such as Fe-Ni-Mg powders as catalyst, the environmental temperature of $600^{\circ} \mathrm{C}$, the arc current of 100A, helium (the purity about $99.995 \%$ ) as buffer gas and the pressure of 500 torr, SWCNTs were synthesized by arc discharging furnace at controlled temperature. The effects of catalyst composition and content on the formation of SWCNTs are mainly investigated.

The Fe-Ni-Mg (5 wt \%) catalyst composition of (wt\%): $2: 1: 0.5,2: 1: 2,2: 2: 1,2: 3: 1$ and $1: 1: 1$ is studied, respectively. The results are shown in Table 1 and Figure 1.

The results from Table 1 show that the effect of catalyst composition on the production and purity of SWCNTs is obvious. With the content of Fe increasing, the production and purity of SWCNTs all decreased. That is to say, low content of Fe is effective to improve the production of SWCNTs. Meanwhile, low content of Mg is not effective to improve the production of SWCNTs, suitable Mg content can improve the production of SWCNTs. Mg has small radius and low evaporation point because the high content of $\mathrm{Mg}$ may increase the pressure of chamber so that the production is decreased. But the low content $\mathrm{Mg}$ cannot exert its action. When the catalyst composition is $2: 1: 2 \mathrm{wt} \%$, the production of SWCNTs $12 \mathrm{~g} / \mathrm{h}$, and the relative SWCNTs purity is $80 \%$. Therefore, the suitable catalyst composition can make the production rate and purity of SWCNTs high. The typical TEM was shown in Figure 1. Figure 1 shows that the SWCNTs have some impurities.

Then we set the condition of Fe-Ni-Mg (2:1:2 wt\%) catalyst content. It is $8,6,5,4,3,2$, and $1 \mathrm{wt} \%$, respectively. The results are shown in Table 2 and Figure 2.

The results from Table 2 show that the effect of catalyst content on the production and purity of SWCNTs is also obvious. With the catalyst content increasing, the production and purity of SWCNTs increase. Meanwhile, the catalyst content result (5\%) is a critical point. This value is, extensive the production and purity of SWCNTs decrease. When the catalyst content is $5 \%$, the production of SWCNTs is 12 grams per hour, and the relative SWCNTs purity is $70 \%$. The typical TEM was shown in Figure 2.

SEM image of this carbon nanotube is shown in Figure 3. HRTEM image of them is shown in Figure 4. The XRD and Raman patterns are shown in Figures 5 and 6.

Figure 3 shows that the purified SWCNTs are very pure and the tubes congregate bundles due to the Van der vaals. HRTEM image of Figure 4 shows that the SWCNTs have high purity and the diameter is about $1.3 \mathrm{~nm}$ by measurement. Generally, an individual carbon tube is difficult to exist by

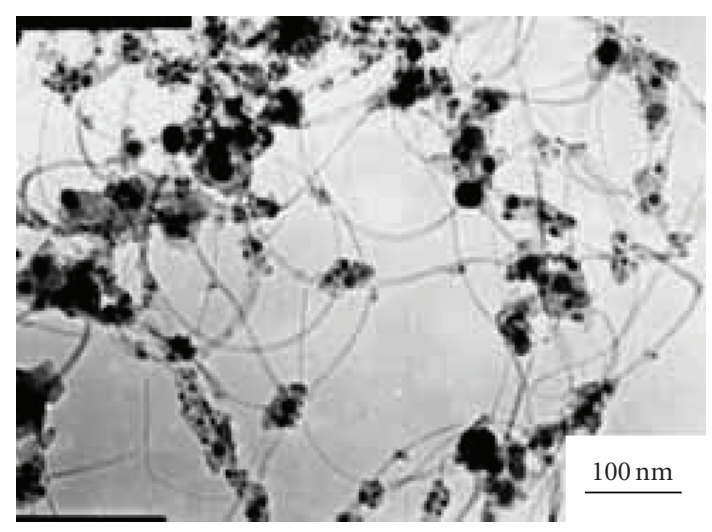

FIgUre 1: TEM image of SWCNTs produced with FeS-Ni-Mg (2 : $1: 2$ ) as catalyst.

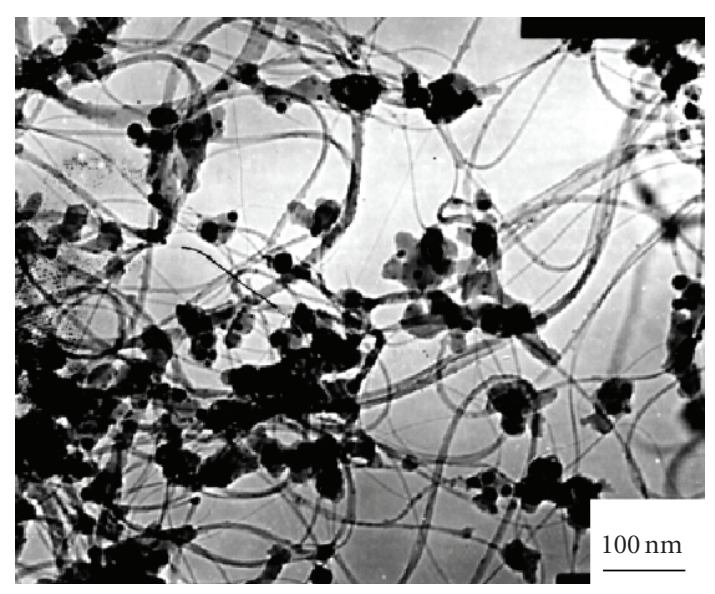

FIGURE 2: TEM image of SWCNTs produced with Fe-Ni-Mg as catalyst (5\%).

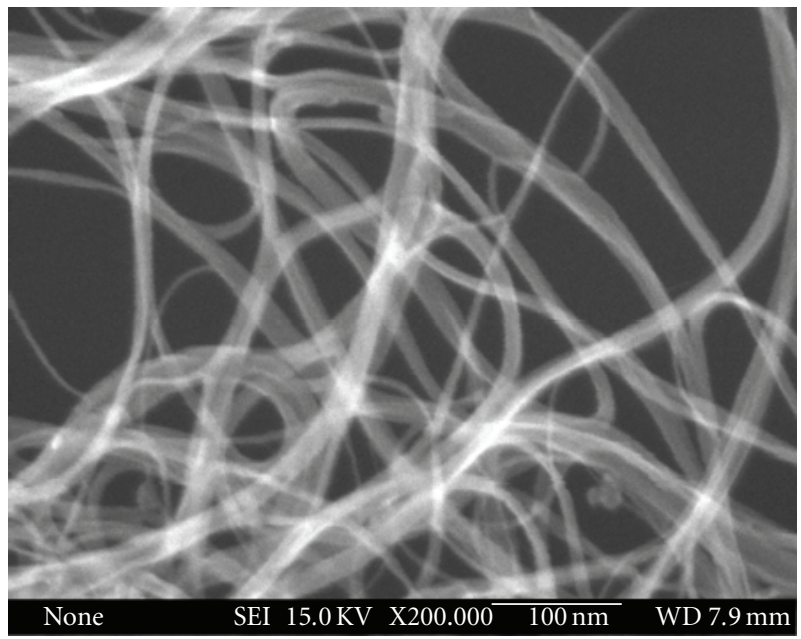

Figure 3: SEM image of purified SWCNTs. 
TABLE 1: The effect of catalyst composition on the production and relative purity of SWCNTs.

\begin{tabular}{c|cc}
\hline Fe-Ni-Mg (5\%) composition/wt\% & production/g/h & (a) Relative SWCNT purity (\%) \\
\hline $2: 1: 2$ & 12 & 70 \\
$1: 1: 1$ & 10 & 70 \\
$2: 1: 1$ & 8.3 & 64 \\
$2: 2: 1$ & 9.7 & 60 \\
$2: 1: 0.5$ & 7.5 & 48 \\
$3: 1: 1$ & 8.5 & 1 \\
$2: 3: 1$ & 5.9 & \\
\hline
\end{tabular}

(a) The relative nanotube purity in sample (a) is higher than sample (b) and has increased by $x \%$.

TABLE 2: The effect of catalyst content on the production and relative purity of SWCNTs.

\begin{tabular}{c|cc}
\hline Fe-Ni-Mg $(2: 1: 2 \mathrm{wt} \%)$ content $(\mathrm{wt} \%)$ & production $(\mathrm{g} / \mathrm{h})$ & (a) Relative SWCNT purity $(\%)$ \\
\hline 8 & 7.6 & 50 \\
6 & 8.9 & 80 \\
5 & 12.1 & 60 \\
4 & 8.8 & 56 \\
3 & 8.5 & 45 \\
2 & 8 & 1 \\
1 & 7.4 & \\
\hline
\end{tabular}

(a) The relative nanotube purity in sample (a) is higher than sample (b) and has increased by $x \%$.

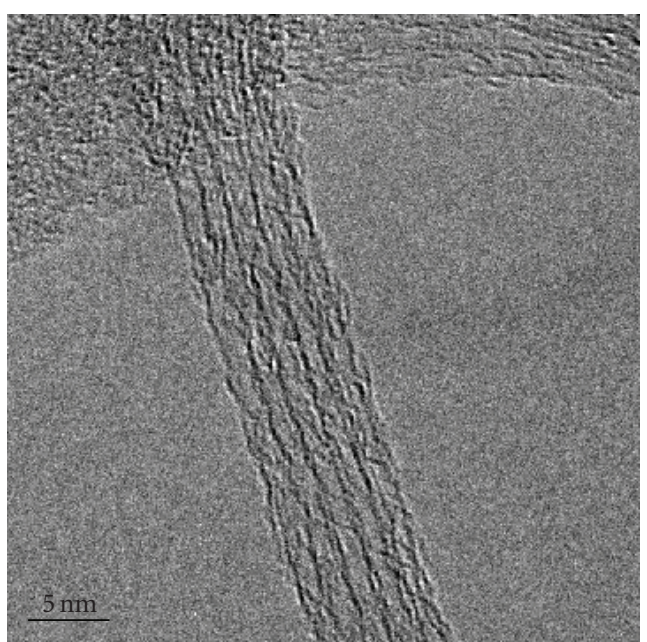

Figure 4: HRTEM image of SWCNTs.

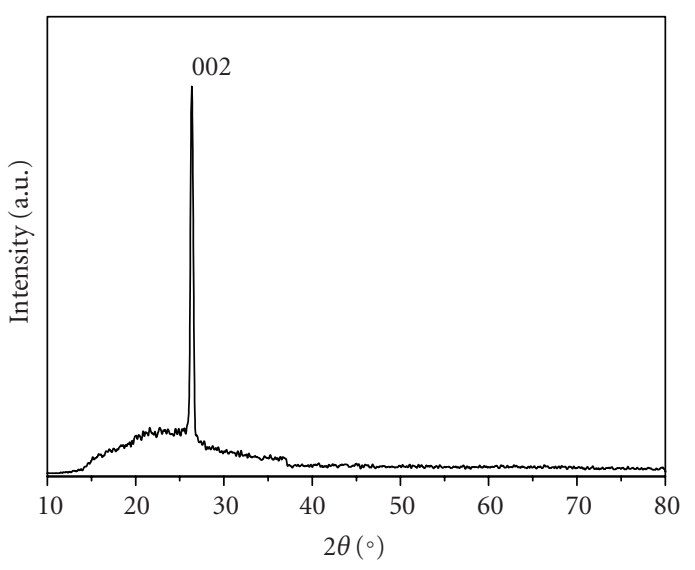

FIGURE 5: XRD pattern of SWCNTs. the affection of electron beam while TEM or HRTEM is carried out. Therefore, a bundle of SWCNT can be seen.

XRD pattern of Figure 5 shows that the diffraction peak of the 002 face at $26.3^{\circ}$ is sharp compared to graphite diffraction peak. The microstructure of SWCNTs has good graphite crystallinity, it is a perfectly hexagonal crystal structure. Raman patterns of Figure 6 show that the SWCNTs have two RBM peaks. The correspondence diameters are 1.38 and $1.22 \mathrm{~nm}$ according to $d=224 / \omega$, the RBM frequency $(\omega)$ about 162 and $183 \mathrm{~cm}^{-1}$, respectively. The D-band(defect band) almost disappear, which reveals that the defective graphite structure is very low. The G-band(graphite band) can be seen at $1592 \mathrm{~cm}^{-1}$, which is a characteristic peak of carbon tube.

The results indicate that the cooperative function of catalyst composition and content play an important role in the production and the purity of SWCNTs. Compared to previous literatures, the present work only exhibits that the same catalyst which has different composition and content lead the different productions. Optimal parameters have been obtained by experimental researches. We think that moderate catalyst composition and content may enhance the dynamics process of the growth of SWCNTs. The further research of the growth mechanism will be carried out. 


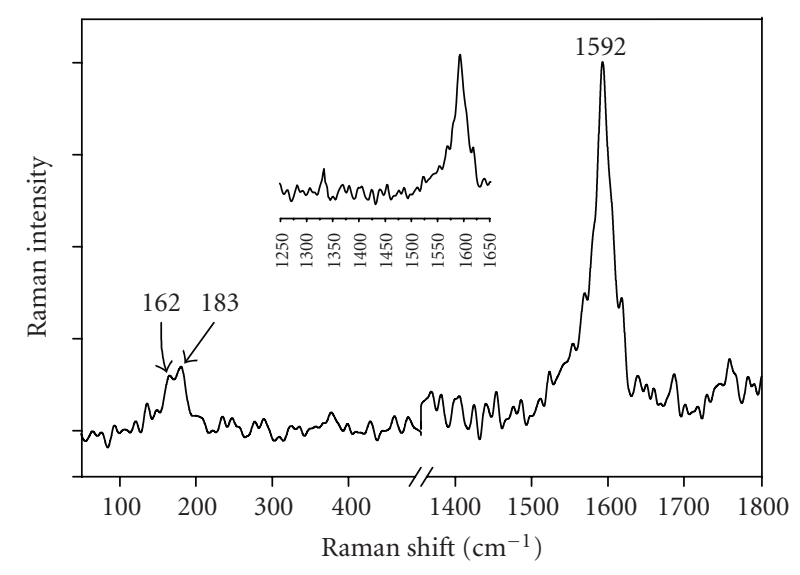

FIGURE 6: Raman pattern of SWCNTs.

\section{CONCLUSION}

SWCNTs were prepared by a modified arc discharge using $\mathrm{Fe}-\mathrm{Ni}-\mathrm{Mg}$ powders as catalyst at $600^{\circ} \mathrm{C}$. The effects of catalyst composition and content on the production rate and purity of SWCNTs are obvious. While the catalyst composition and content are $2: 1: 2 \mathrm{wt} \%$ and $5 \mathrm{wt} \%$, respectively, the production rate of SWCNTs is 12 grams per hour, and the purity of SWCNTs is about 70\%. And the diameter of SWCNTs is $1.22 \sim 1.38 \mathrm{~nm}$.

\section{ACKNOWLEDGMENT}

This work was financially supported by China Postdoctoral Science Foundation (Grant no. 20060391006).

\section{REFERENCES}

[1] S. Iijima and T. Ichihashi, "Single-shell carbon nanotubes of 1nm diameter," Nature, vol. 363, no. 6430, pp. 603-605, 1993.

[2] D. S. Bethune, C. H. Kiang, M. S. De Vries, et al., "Cobaltcatalysed growth of carbon nanotubes with single-atomiclayer walls," Nature, vol. 363, no. 6430, pp. 605-607, 1993.

[3] M. S. Dresselhaus, G. Dresselhaus, and P. C. Eklund, Science of Fullerenes and Carbon Nanotubes, Academic Press, New York, NY, USA, 1996.

[4] T. W. Ebbesen, Carbon Nanotubes: Preparation and Properties, CRC Press, Boca Raton, Fla, USA, 1997.

[5] P. M. Ajayan, "Nanotubes from carbon," Chemical Reviews, vol. 99, no. 7, pp. 1787-1800, 1999.

[6] T. Zhao and Y. Liu, "Large scale and high purity synthesis of single-walled carbon nanotubes by arc discharge at controlled temperatures," Carbon, vol. 42, no. $12-13$, pp. 27652768, 2004.

[7] T. Zhao and Y. Liu, "Large scale synthesis of single-walled carbon nanotubes by an arc-discharge method at controlled temperatures," Acta Physica Sinica, vol. 53, no. 11, pp. 340-345, 2004.

[8] C. Journet, W. K. Maser, P. Bernier, et al., "Large-scale production of single-walled carbon nanotubes by the electric-arc technique," Nature, vol. 388, no. 6644, pp. 756-758, 1997.
[9] A. Thess, R. Lee, P. Nikolaev, et al., "Crystalline ropes of metallic carbon nanotubes," Science, vol. 273, no. 5274, pp. 483-487, 1996.

[10] M. Su, B. Zheng, and J. Liu, "A scalable CVD method for the synthesis of single-walled carbon nanotubes with high catalyst productivity," Chemical Physics Letters, vol. 322, no. 5, pp. 321326, 2000.

[11] R. L. Vander Wal and L. J. Hall, "Flame synthesis of Fe catalyzed single-walled carbon nanotubes and Ni catalyzed nanofibers: growth mechanisms and consequences," Chemical Physics Letters, vol. 349, no. 3-4, pp. 178-184, 2001.

[12] T. Guo, P. Nikolaev, A. Thess, D. T. Colbert, and R. E. Smalley, "Catalytic growth of single-walled nanotubes by laser vaporization," Chemical Physics Letters, vol. 243, no. 1-2, pp. 49-54, 1995.

[13] B. P. Tarasov, V. E. Muradyan, Y. M. Shul'ga, et al., "Synthesis of carbon nanostructures by arc evaporation of graphite rods with Co-Ni and $\mathrm{YNi}_{2}$ catalysts," Carbon, vol. 41, no. 7, pp. 1357-1364, 2003.

[14] M. Yudasaka, N. Sensui, M. Takizawa, S. Bandow, T. Ichihashi, and S. Iijima, "Formation of single-wall carbon nanotubes catalyzed by Ni separating from $\mathrm{Y}$ in laser ablation or in arc discharge using a C target containing a NiY catalyst," Chemical Physics Letters, vol. 312, no. 2-4, pp. 155-160, 1999.

[15] T. Zhao, "Large scale and high purity synthesis of carbon nanotubes by temperature controlled arc discharge and investigation on their growth mechanism," Doctoral dissertation, Xi'an Jiaotong University, Xi'an, China, 2005. 

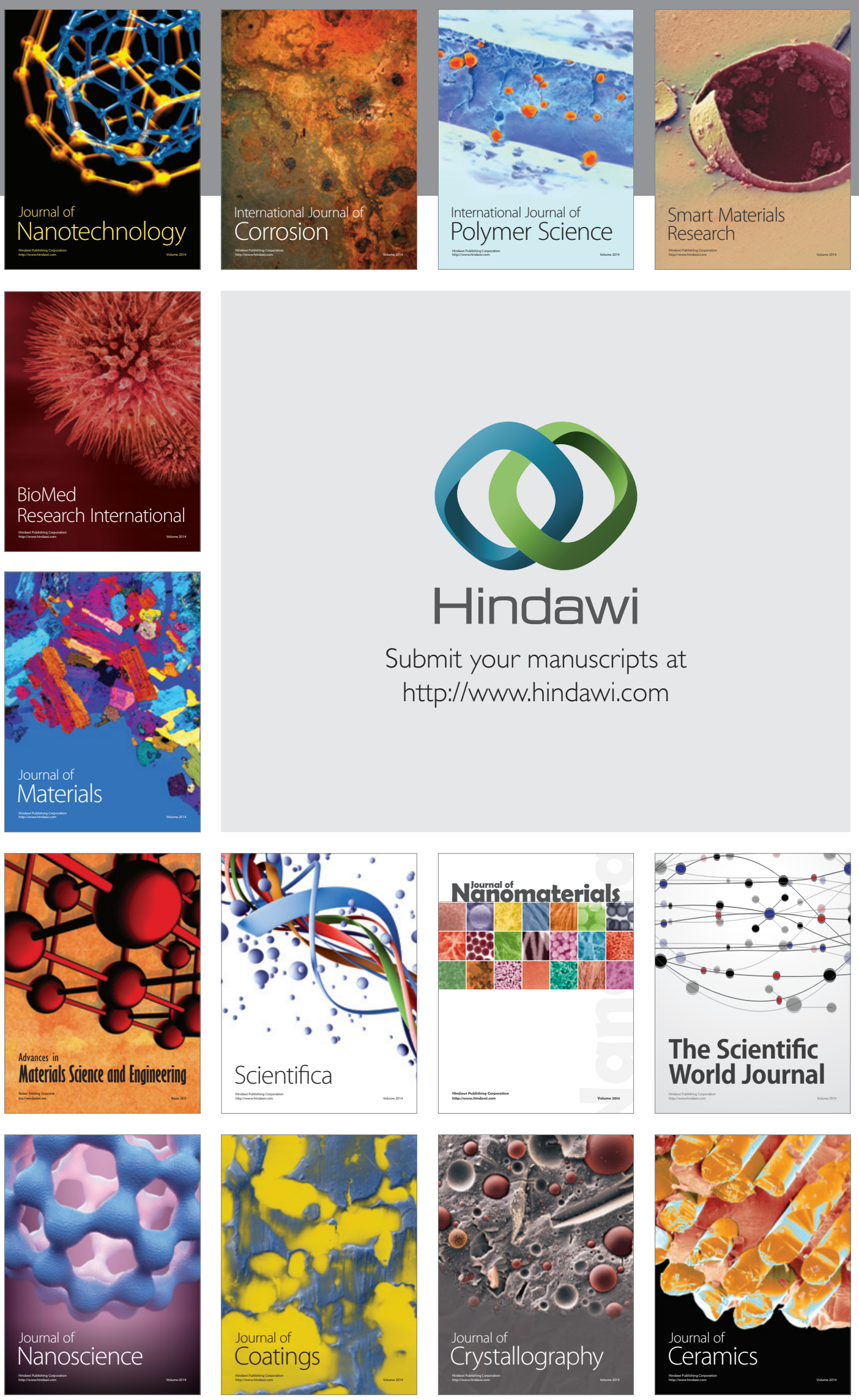

The Scientific World Journal

Submit your manuscripts at

http://www.hindawi.com

\section{World Journal}

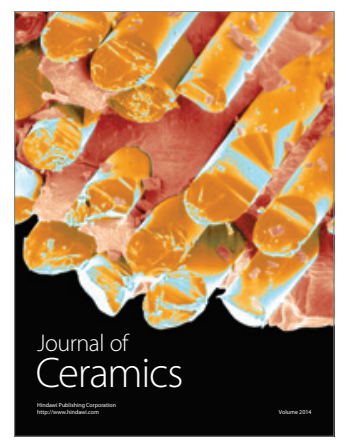

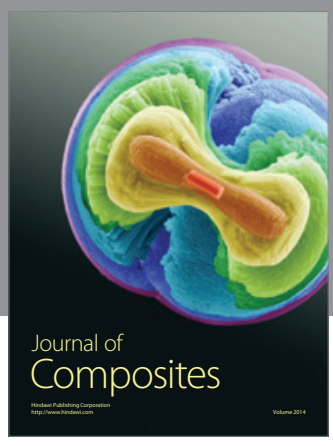
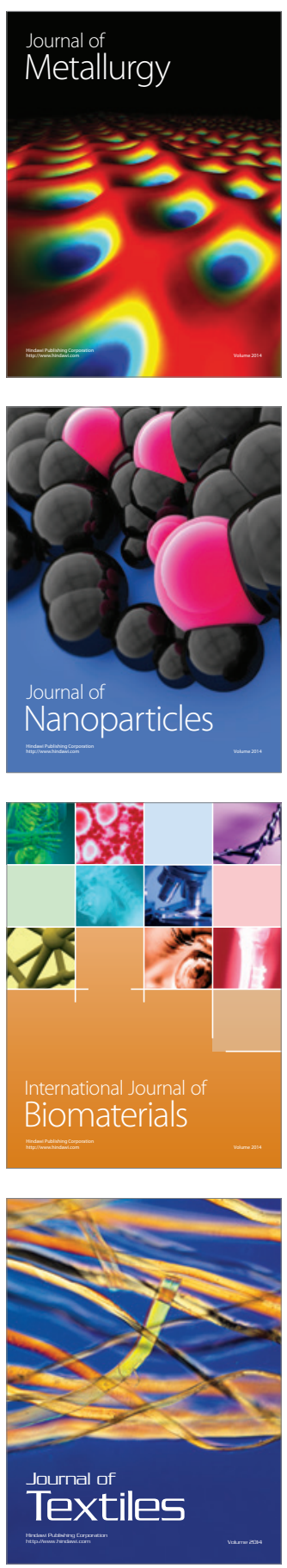